\title{
¿El expediente clínico debe ser clasificado como confidencial y reservado?
}

\author{
Juana Arellano-Mejía, ${ }^{*} \bowtie$ Carlos Augusto Sánchez-Morales ${ }^{*}$
}

*Instituto Nacional de Enfermedades Respiratorias Ismael Cosío Villegas;

¥Instituto Nacional de Ciencias Médicas y Nutrición «Salvador Zubirán».

Trabajo recibido: 10-XI-2016; aceptado: 23-II-2017

\begin{abstract}
RESUMEN. La confidencialidad y tratamiento adecuado de los datos personales de los pacientes son hábiles en virtud de lo dispuesto en los ordenamientos legales en México hasta el momento existentes y que se fusionan con los conceptos de bioética aplicables al ámbito público, en específico al Sistema Nacional de Salud. Estos elementos convergen de manera definitiva con los derechos humanos más entrañables y largamente pugnados por la humanidad como son el derecho a la intimidad y la vida privada. En el presente artículo se destaca una línea de investigación documental y descriptiva, la clasificación del expediente clínico y el panorama del acceso a datos personales en la Administración Pública Federal, para exhibir las dos opciones de clasificación: la reservada y la confidencial en temas no concluyentes del expediente clínico. Se propone un modelo y/o criterio útil para todas las instituciones de la Administración Pública Federal respecto al resguardo y manejo del expediente clínico con especial atención de los procesos y/o procedimientos inconclusos, como son el estado de necesidad terapéutico, pronósticos y/o diagnósticos, sin olvidar todos los datos personales en él vertidos.
\end{abstract}

Palabras clave: Expediente clínico, confidencialidad, reserva, datos personales.

ABSTRACT. Confidentiality and due care of personal data to be successful must comply with all existing legal instruments in Mexico, moreover ethics principals that converges with rights of access and correction of personal data, should be observed because they are all binding to the public field, but specifically to the national health system, those elements mentioned before are intimately related to the most deep defended and valuable human rights along history like, intimacy and private life. This review article emphasize one line of documental and descriptive investigation about the medical record and its classification, and shows a brief outlook of personal data access in Mexican public administration. The purpose here is to show both available options of classification: confidential and reserved, that can apply in specific cases like unsolved matters which are contained in the medical record. At last, but not at least (as a conclusion) is shown a proposal of a model or criteria useful for all the institutions focused in health that are part of the public administration, with highlights in due care of medical records where unsolved matters are contained, that can be identified like prognosis and diagnosis.

Key words: Medical record, confidentiality, reserve, personal data, due care.

\section{Abreviaturas:}

APF = Administración Pública Federal.

LFTAIPG = Ley Federal de Transparencia y Acceso a la

Información Pública Gubernamental.

LGT = Ley General de Transparencia y Acceso a la Información Pública.

\section{INTRODUCCIÓN}

El acceso a datos personales (DP), es un derecho elevado a rango constitucional que permite acceder y reproducir la información para la identificación de una persona, la cual debe considerarse como confidencial, contenida y/o depositada en una entidad o dependencia de la Administración Pública Federal (APF). Más aún, es necesario decir que existe la posibilidad de corregir
$\mathrm{DS}=$ Documento de Seguridad.

$\mathrm{SDP}=$ Sistemas de Datos Personales.

IFAI = Instituto Federal de Acceso a la Información Pública y Protección de Datos Personales.

INAI = Instituto Nacional de Acceso a la Información Pública y Protección de Datos Personales.

$\mathrm{SO}=$ Sujetos obligados .

tales datos según se advierte en el contenido de la Ley Federal de Transparencia y Acceso a la Información Pública Gubernamental (LFTAIPG.)

¿Qué debe entenderse por DP? en este caso hay que remitirse a la fracción II del Artículo 3 de la LFTAIPG, la cual establece que éstos se entenderán como cualquier información concerniente a una persona identificada o identificable y que puede estar contenida en sistemas físicos o automatizados. 
Ahora bien, conviene decir que la LFTAIPG establece que el acceso a DP es un derecho que le corresponde únicamente al titular de los datos. Sin embargo, se prevén algunas excepciones para que ese derecho se ejercite por terceros autorizados mediando en todos los casos un consentimiento expreso. Esto es posible a través de un poder con carácter legal, según indica el ordenamiento de la materia. Otra excepción se da en caso de que la expresión de la voluntad del titular de los datos no pudiera ser otorgada; entonces, el acceso y/o la corrección de los DP puede lograrse en virtud de la acreditación del vínculo consanguíneo o conyugal.

El acceso a los DP no sólo está relacionado con las medidas que los sujetos obligados (SO [entiéndase entidades o dependencias de la APF]) adopten para que verdaderamente sea éste un derecho personalísimo, también está relacionado con todas las acciones que se realizan para asegurar que la información confidencial sea de acceso restringido.

Las medidas de protección a la información personal que los SO poseen se encuentran en el denominado Documento de Seguridad (DS) que los comités de transparencia (CT) de la APF deben elaborar en virtud de los ordenamientos legales aplicables.

Conviene destacar que el CT es un órgano colegiado al interior de los SO, el cual en pocas palabras vigila y propone medidas y acciones tendientes a lograr el adecuado cumplimiento de los ordenamientos legales relativos a estos temas.

Así, el DS debe enunciar cada uno de los Sistemas de Datos Personales (SDP) que obren en poder y custodia de los SO detallando la ubicación de la información, la forma en que se resguarda, el tiempo de conservación, el nombre de las personas que acceden a ellos y el nombre (s) del (los) responsable (s) de la guarda y custodia de la información, entre otras.

Por otro lado, las acciones que en materia de protección de DP se desarrollen en los SO no pueden soslayarse, pues se trata de medidas que tienen por objetivo la protección de seres humanos. Situación de especial importancia, pues salvaguardar y tratar adecuadamente la información e intimidad de las personas es fundamental para sus depositarios.

En México, el tema de DP para el ámbito gubernamental está considerado en la LFTAIPG y su Reglamento, así como en otros ordenamientos legales. Sin embargo, sabiendo que los excesos y/o defectos son dables, se presentan elementos, argumentos, opiniones y/o conclusiones que conformarán una propuesta para mejorar la situación actual respecto al manejo de los DP.

\section{ANTECEDENTES}

Lo mencionado anteriormente tuvo sus orígenes en Suecia en el año 1766. En México fue hasta 1977, es decir, más de doscientos años después cuando se incorporó al texto legal de máxima importancia en el Artículo $6^{\circ}$ constitucional, el cual se cita para hacer patente el dicho «El derecho a la información será garantizado por el Estado». Este texto y su trasfondo como señaló Carpizo, tuvo impacto en la manipulación de la información permitiendo en el ideal que ésta fuera objetiva y no se deformara, lo que atraería opciones a la decisión política del ciudadano para enriquecer su propia existencia. ${ }^{1,2}$ Años más tarde se creó la LFTAIPG, el Reglamento de la LFTAIPG, y los Lineamientos de Protección de Datos personales.

Es importante mencionar que desde 1977 al 2015, la Constitución Política de los Estados Unidos Mexicanos ha tenido diversas modificaciones; aunque han existido largos períodos de inactividad por parte del Estado para la atención de estos temas. Pero poco a poco fue cobrando fuerza real hasta hacer patente la equiparación de este derecho de acceso y la protección a la información con la protección a la salud o la recepción de educación.

Sin embargo, el proceso no ha sido simple pero la intención de mejorar no cesa y es mucho más tangible ahora que se conocen y aplican más elementos en este sentido. De esa suerte y por el esfuerzo continuo y continuado respecto a estos temas han sido identificadas las áreas de oportunidad como una manera de mejoramiento, p. ej. la protección de los DP a través de una doble clasificación de la información contenida en los expedientes clínicos, resguardados por las instituciones de salud del Sector Público.

\section{EL PROBLEMA}

El acceso a los DP en las instituciones de salud está íntimamente relacionado con el documento madre de la atención médica denominado: expediente clínico, donde se asientan los aspectos relevantes del estado de salud de una persona y se sintetiza y expresa la relación médico-paciente.

El expediente, además, es el elemento fundamental con el que se acredita el cumplimiento en materia de salud del Plan Nacional de Desarrollo 2013-2018, que tiene como eje medular «proporcionar servicios eficientes, cálidos y seguros para el paciente». ${ }^{3} \mathrm{El}$ cumplimiento de la Norma Oficial Mexicana (NOM) 004SSA-3-2012 para el manejo del expediente y de la LFTAIPG obligan a proteger los DP contenidos en expedientes clasificados como "confidenciales», lo 
que agrega un elemento concerniente a la seguridad para el paciente, de conformidad con los Criterios de Certificación Hospitalaria emitidos por el Consejo de Salubridad General.

El expediente clínico es uno de los elementos centrales de la materia de acceso, el cual de suyo se entiende como confidencial, en virtud de contener información como es el estado de salud físico y/o mental de una persona.

De acuerdo a la NOM, el expediente clínico es propiedad de la institución que lo genera y conserva; sin embargo, la información plasmada en él únicamente corresponde al aportante, es decir, al paciente. Aunque algunos centros hospitalarios consideraban como suya la información contenida en el expediente clínico y ello generaba reticencia para lograr el procedimiento de acceso a la información.

Para el año 2004, la posición de muchas instituciones de salud pública ante la contundente apertura del expediente clínico fue de asombro e incredulidad, pues tal apertura daba la oportunidad de otorgar acceso a un documento que tradicionalmente era manejado como «inaccesible». En ese entonces, aun cuando era evidente que el acceso no estaría nunca más condicionado (salvo situaciones específicas) la resistencia era definitiva, provocando en años subsecuentes múltiples negativas respecto al acceso al expediente clínico. Ante tal evento, el entonces Instituto Federal de Acceso a la Información y Protección de Datos (IFAI) hoy Instituto Nacional de Acceso a la Información Pública y Protección de Datos Personales (INAI) ordenó a distintas instituciones de salud el acceso al expediente, con el apercibimiento definitivo para el caso de incumplimiento de dar vista e intervención a los Órganos Internos de Control correspondientes, lo que implicaría en su caso responsabilidad administrativa que podría traer la destitución e inhabilitación de quien se determinara responsable.

Al día de hoy, las negativas de acceso al expediente se han reducido, pues las interpretaciones que en su momento existieron dieron pie a que el IFAI emitiera el siguiente «CRITERIO/0004-09 Expediente clínico. Por regla general su confidencialidad no es oponible al titular de los datos personales o a su representante legal».

Aun con lo anterior, la posición de un gran número de médicos mexicanos respecto al acceso al expediente clínico persiste, generando un clima de incomodidad y resistencia para satisfacer el procedimiento de acceso. Esta situación, entre otras, son motivo para analizar las causas del pensamiento del grupo médico y reflexionar sobre la conveniencia del acceso al expediente clínico. En este sentido se fórmula una propuesta de clasificación del expediente con base en la temporalidad y definitividad de los elementos que se encuentran vertidos en el mismo.

Con este antecedente se realiza el siguiente análisis breve de las leyes del país. Por orden jerárquico en primer lugar está la Constitución Política de los Estados Unidos Mexicanos que en su Artículo $6^{\circ}$ cita: «...El derecho a la información será garantizado por el Estado....toda persona tiene derecho al libre acceso a información...». Estos elementos son la antesala para adentrarse en este tema, pues hace patente la oportunidad sin límite para acceder a la información.

Se aporta una visión amplia de lo que este derecho a la información implica y cómo poder llegar al acceso de DP, en específico a los que contiene el expediente clínico. En ese sentido citamos:

«...A. Para el ejercicio del derecho de acceso a la información, la Federación, los Estados y el Distrito Federal, en el ámbito de sus respectivas competencias, se regirán por los siguientes principios y bases: ...II. La información que se refiere a la vida privada y los datos personales será protegida en los términos y con las excepciones que fijen las leyes».

Este elemento correspondiente al ejercicio del derecho de acceso a la información de manera clara, establece un límite tratándose de su ejercicio, lo cual permite la nitidez necesaria en este recorrido cognoscitivo para llegar a la clasificación de la información contenida en el expediente. En otro fragmento de la constitución se cita: «...La ley establecerá aquella información que se considere reservada o confidencial». Con esto se vislumbra la necesidad de recurrir a un nuevo instrumento de menor jerarquía, el cual contiene elementos más tangibles y especializados la LFTAIPG. Aun y cuando todo lo que contiene esta ley es importante, lo que resulta de interés elemental son los supuestos de clasificación, pues son la base de nuestra propuesta.

A manera de ejemplo tenemos:

Plutarco Calles con domicilio en Avenida Vasco de Quiroga, Colonia Sección XVII. C.P 14020, tiene 29 años y es casado.

Todos los datos en este ejemplo corresponden a Plutarco, quien es una persona física, al hacerlo identificable motiva que estos datos se consideren personales y por lo tanto confidenciales.

Corresponde ahora en orden de ideas y correlación referir el supuesto actualizable para la clasificación de la información como confidencial, la cual es efectiva naturalmente en virtud de lo siguiente.

Artículo 18. Como información confidencial se considerará: 
II. Los datos personales que requieran el consentimiento de los individuos para su difusión, distribución o comercialización en los términos de esta ley.

Para el Sector Salud, es simple ver que el contenido y/o elementos que componen el expediente clínico encuadran claramente en la hipótesis normativa plasmada arriba, en virtud de que la información contenida en dicho expediente hace definitivamente identificable a la persona que en este caso se considera paciente.

Por otro lado, el contenido del Artículo 24 de la misma ley, hilado con lo previo permite entender que existe una oportunidad para acceder a los datos con sus limitantes correspondientes, lo cual beneficia el acceso a la información, sin desproteger la vida privada y los DP. Para pronta referencia se cita un fragmento del último artículo referido.

«Sin perjuicio de lo que dispongan otras leyes, sólo los interesados o sus representantes podrán solicitar a una unidad de enlace o su equivalente, previa acreditación, que les proporcione los datos personales que obren en un sistema de datos personales».

Este último supuesto hace patente una limitante parcial para acceder a los DP contenidos en los archivos del ámbito público, siempre que la solicitud de acceso se logre por medios físicos a través de la Unidad de Transparencia, pues de esa forma se ajusta a la hipótesis normativa y es posible la comprobación de la personalidad de los interesados o sus representantes. No así tratándose del medio electrónico determinado para tales efectos INFOMEX (hoy Plataforma Nacional de Transparencia), pues en ese medio se puede intentar el acceso a tales datos sin la acreditación de la personalidad; en tal caso, depende de la habilidad y el conocimiento de los integrantes de la Unidad de Transparencia para hacer efectivo el límite de acceso a los DP en virtud de la probable usurpación o suplantación de la identidad. Importante tarea, pues aun y cuando el ordenamiento legal que protege y limita los derechos a los que nos hemos referido determinan una serie de requisitos formales para acceder al tipo de información que nos ocupa, el mismo sistema INFOMEX es omiso en su exigencia. Sin embargo, la idea principal y de la que se pretende entendimiento y claridad es que los datos personales contenidos en el expediente clínico se ajustan a la hipótesis normativa que permite clasificarlos como información Confidencial de forma innegable.

Ya claros en que la información que se contiene en el expediente se ajusta a los supuestos de confidencialidad, resulta importante encuadrar una segunda clasificación que es justamente el tema toral de este instrumento; en este caso nos referimos a la clasificación como: Reservada.

Para hacer simple el evento se acude nuevamente al contenido de la LFTAIPG la que en su Artículo 13 establece que: «Como información reservada podrá clasificarse aquélla cuya difusión pueda: IV. Poner en riesgo la vida, la seguridad o la salud de cualquier persona»; ésta se concatena con la fracción VI que establece: La que contenga las opiniones, recomendaciones o puntos de vista que formen parte del proceso deliberativo de los servidores públicos, hasta en tanto no sea adoptada la decisión definitiva, la cual deberá estar documentada.

Para el caso del expediente, las hipótesis referidas operan y son hábiles porque en el momento de la atención médica existen apreciaciones subjetivas; esto es, anotaciones no concluyentes que después de ser robustecidas y comprobadas con otros tantos eventos permitirán en su conjunto determinar un diagnóstico. Sin embargo, dicho diagnóstico no es definitivo, es decir, es presuncional.

V. gr:: Plutarco Calles acude al médico, éste después de una revisión y la interpretación de los estudios que se le indicaron previamente, advierte que los síntomas de Plutarco empatan con los que corresponden a las personas que padecen cáncer, lo plasma en el expediente sin comentarlo con Plutarco pues necesitaría mayores elementos para poder concluir sin equívocos respecto de su impresión diagnóstica. Sin embargo, si el paciente decide ejercitar el derecho de acceso correspondiente y no se hace efectiva la segunda clasificación Reservada que proponemos y el expediente se transmite, puede causarle una impresión fatal con la simple lectura. Esto comprueba sin lugar a dudas que el supuesto es efectivo y que por lo tanto la propuesta es viable y oportuna.

Es necesario tener claridad de que en muchos momentos el expediente clínico deberá adquirir la clasificación como Reservado de manera ordinaria, en virtud de las opiniones, recomendaciones o puntos de vista de los médicos tratantes, ya que claramente el proceso de determinación se integra con todas las aseveraciones en él vertidas por el o los médicos tratantes, sin ser éstas definitivas. De suerte que los excesos pueden ocurrir si es que se permite el acceso a dicha información de forma bidireccional, es decir, en perjuicio del paciente y/o de los médicos tratantes.

Además de lo anterior, la doble clasificación del expediente clínico parece aún más natural a la vista de lo dispuesto en el Vigésimo Cuarto de los Lineamientos para la Clasificación y Desclasificación de la Información emitidos por el IFAI el cual dispone: Para los efectos de la fracción VI del Artículo 14 de la Ley, se considerará 
que se ha adoptado la decisión definitiva cuando el o los servidores públicos responsables de tomar la última determinación resuelvan el proceso deliberativo de manera concluyente, sea o no susceptible de ejecución.

De esto debe entenderse que las opiniones y/o comentarios subjetivos que se plasman en el expediente clínico hasta el o los pronósticos, deberán de ser reservados en virtud del carácter no concluyente en el que se encuentran.

De los ordenamientos de más reciente publicación que son las Leyes General y Federal de Acceso a la Información Pública, se advierte la exclusión de los elementos que corresponden al acceso a DP, pues la intención es la creación de instrumentos normativos correspondientes únicamente para regular y definir tal materia, sin modificaciones radicales en su contenido.
Esto es acertado, pues debe realizarse la separación tratándose de información confidencial y/o reservada para aquellos elementos que por su delicadeza, importancia y algidez deban permanecer de alguna forma restringidos para su publicación o publicidad. De esa suerte y con la intención de agotar el evento se enuncian los supuestos normativos pares con el tema de interés en la tabla 1.

Un elemento más es añadido a estos dos ordenamientos legales para darle habilidad a la clasificación como reservada: la prueba de daño.

\section{EL EXPEDIENTE CLÍNICO}

Con la intención de comprender la propuesta que se plantea, hay que referirse al concepto de expediente

Tabla 1. Supuestos normativos.

Ley General de Transparencia y Acceso a la Información Pública

Artículo 113. Como información reservada podrá clasificarse aquélla cuya publicación: $\mathrm{V}$. Pueda poner en riesgo la vida, seguridad o salud de una persona física; VIII. La que contenga las opiniones, recomendaciones o puntos de vista que formen parte del proceso deliberativo de los servidores públicos, hasta en tanto no sea adoptada la decisión definitiva, la cual deberá estar documentada;

III. Las que por disposición expresa de una ley tengan tal carácter, siempre que sean acordes con las bases, principios y disposiciones establecidos en esta Ley y no la contravengan; así como las previstas en tratados internacionales

Artículo 114. Las causales de reserva previstas en el artículo anterior se deberán fundar y motivar, a través de la aplicación de la prueba de daño a la que se hace referencia en el presente Título
Ley Federal de Transparencia y Acceso a la Información Pública

Artículo 110. Conforme a lo dispuesto por el artículo 113 de la Ley General, como información reservada podrá clasificarse aquélla cuya publicación:

V. Pueda poner en riesgo la vida, seguridad o salud de una persona física; VIII. La que contenga las opiniones, recomendaciones o puntos de vista que formen parte del proceso deliberativo de los Servidores Públicos, hasta en tanto no sea adoptada la decisión definitiva, la cual deberá estar documentada

XIII. Las que por disposición expresa de una ley tengan tal carácter, siempre que sean acordes con las bases, principios y disposiciones establecidos en la Ley General y esta Ley y no las contravengan; así como las previstas en tratados internacionales

Artículo 111. Las causales de reserva previstas en el artículo anterior se deberán fundar y motivar, a través de la aplicación de la prueba de daño a la que se refiere el artículo 104 de la Ley General

Artículo 104. En la aplicación de la prueba de daño, el sujeto obligado deberá justificar que:

I. La divulgación de la información representa un riesgo real, demostrable e identificable de perjuicio significativo al interés público o a la seguridad nacional;

II. El riesgo de perjuicio que supondría la divulgación supera el interés público general de que se difunda, y III. La limitación se adecua al principio de proporcionalidad y representa el medio menos restrictivo disponible para evitar el perjuicio

Artículo 116. Se considera información confidencial la que contiene datos personales concernientes a una persona identificada o identificable. ${ }^{i}$
Artículo 113. Se considera información confidencial:

I. La que contiene datos personales concernientes a una persona física identificada o identificable;ii

' Ley General de Transparencia y Acceso a la Información Pública, Accesible en http://www.diputados.gob. mx/LeyesBiblio/ pdf/LGTAIP. Pdf (Consultado el 28 de enero de 2017).

ii Ley Federal de Transparencia y Acceso a la Información Pública. Accesible en http://www.dof.gob.mx/nota_detalle.php ? codigo =5436283\&fecha $=09 / 05 / 2016$ (Consultado el 20 de enero de 2017). 
clínico (de acuerdo a la doctrina y la práctica médica) como el documento que evidencia la relación médicopaciente y como el eje del quehacer médico.

En el marco de la atención y la enseñanza médica, fueron necesarias nuevas medidas de control y organización de la información médica.

«En esta vocación de servicio que es la medicina, surge como una necesidad para plasmar con la palabra escrita los hechos, el expediente clínico. El Expediente Clínico es el documento resultante de la entrevista médico-paciente, y la bitácora de cada individuo hospitalizado, el expediente guarda los datos clínicos ordenados, y es una especie de inventario contenido en una minuta, de cada caso clínico que llega al hospital, o de cada paciente al ingresar al consultorio, y en él, se van acumulando las opiniones diagnósticas de quienes participan en su elaboración». ${ }^{4}$

En el año de 1986 la Secretaría de Salud publicó la Norma Técnica 52 para la elaboración, integración y uso del expediente clínico que estuvo en vigor hasta 1993, año en que se publica la Ley Federal sobre Metrología y Normalización. Esta ley deja sin vigencia las Normas Técnicas del Gobierno Federal, sustituyéndolas por Normas Oficiales Mexicanas.

Después de recibir la opinión científica de las instituciones públicas, privadas y sociales en materia de prestación de servicios de salud, a fin de sistematizar, homogeneizar y actualizar el manejo del expediente que contiene los registros de los elementos técnicos esenciales relativos al estudio racional y la solución de los problemas de salud del usuario (involucrando acciones preventivas, curativas y rehabilitatorias), el 30 de septiembre de 1998 se publicó la NOM 168SSA-1 del manejo del Expediente Clínico, la cual se constituyó como una herramienta de obligatoriedad para los sectores público, social y privado del Sistema Nacional de Salud.

Esta norma recién modificada es hoy la denominada NOM para el Manejo del Expediente Clínico 004SSA3-2012, la cual representa el instrumento para la regulación del expediente clínico y orienta al desarrollo de una cultura de calidad.

En virtud de que el expediente clínico es la base más cercana a la fuente de información y en él se proporcionan las bases del diagnóstico y la consistencia del trabajo del médico y de todo aquel material donde se delineará aquel programa de estudio o tratamiento para un caso específico; éste se elige como el instrumento médico, administrativo, legal educativo y de investigación, de uso obligatorio en todo el terri- torio nacional. También es un adminículo con el que se pretende mejorar la calidad de la atención de los servicios de salud.

En la situación actual de la práctica de la medicina, el expediente clínico es un instrumento para la defensa legal del médico. ${ }^{3}$ Es importante decir que la NOM se conforma de 15 rubros generales, donde destacan por lo menos ocho momentos en los que el diagnóstico y/o pronóstico deben encontrarse plasmados.

\section{SITUACIÓN ACTUAL DEL EJERCICIO DEL DERECHO DE ACCESO AL EXPEDIENTE CLÍNICO EN LA ADMINISTRACIÓN PÚBLICA FEDERAL}

Según el Informe Anual de Labores 2015 emitido por el INAI, las instituciones con el mayor número de solicitudes de información a datos personales son: Instituto Mexicano del Seguro Social (IMSS), Instituto de Seguridad Social de los Trabajadores al Servicio del Estado (ISSSTE), Instituto Nacional de Cardiología (INC) «lgnacio Chávez», Instituto Nacional de Ciencias Médicas y Nutrición (INCMN) «Salvador Zubirán» e Instituto Nacional de Enfermedades Respiratorias (INER) Ismael Cosío Villegas. Al no especificar a qué rubro de DP atienden, los que hoy sustentan el presente documento solicitamos vía INFOMEX a esas instituciones la información respecto del tema más recurrente en ese tópico, encontrando que todas ellas son concernientes al acceso al expediente clínico (tabla 2).

Los hallazgos demuestran que para el período 20112015 se observó un incremento de solicitudes de acceso al expediente clínico de entre el 10 y $15 \%$ por año, siendo el IMSS, ISSSTE, INC «Ignacio Chávez», INNCM «Salvador Zubirán» e INER Ismael Cosío Villegas, las cinco instituciones de salud que atienden el mayor número de solicitudes de información correspondientes a DP y en especial al expediente clínico. Sin embargo, es bueno mencionar que ante una política de austeridad las copias del expediente clínico ocasionan un gasto importante a las instituciones de salud, situación que no siempre es bien recibida máxime que el acceso a información concerniente a DP es gratuita y se otorga en copia simple de acuerdo con lo establecido en la LFTAIPG.

El cumplimiento al acceso a la información del expediente clínico aún genera reticencias al interior de las instituciones de salud, dichas instituciones en su defensa argumentan:

1) El cobro por reproducción de material. Situación imposible de atender al imperar la gratuidad de la 
Tabla 2. Acceso a datos personales sector salud.

\begin{tabular}{|c|c|c|c|c|c|c|}
\hline $\begin{array}{l}\text { Institución/núm. de sol. datos } \\
\text { personalesii }\end{array}$ & 2011 & 2012 & 2013 & 2014 & 2015 & Total \\
\hline INER & 142 & 135 & 164 & 156 & 171 & 768 \\
\hline IMSS & 18,774 & 26,948 & 26,070 & 17,512 & 20,085 & 10,939 \\
\hline ISSSTE & 1,338 & 1,663 & 3,387 & 2,306 & * & 8,694 \\
\hline INCICH & 298 & 602 & 1,217 & 1,305 & * & 3,422 \\
\hline INCMNSZ & 100 & 197 & 364 & 575 & 434 & 1,670 \\
\hline $\begin{array}{l}\text { Institución/núm. de sol. exp. } \\
\text { clínicos }\end{array}$ & 2011 & 2012 & 2013 & 2014 & 2015 & Total \\
\hline INER & 118 & 104 & 138 & 134 & 141 & 635 \\
\hline IMSS & 6,250 & 6,387 & 7,717 & 2,834 & 1,500 & 24,688 \\
\hline ISSSTE & 1,338 & 1,663 & 3,387 & 2,306 & * & 8,694 \\
\hline INCICH & 265 & 518 & 993 & 643 & 303 & 2,722 \\
\hline INCMNSZ & 50 & 81 & 141 & 230 & 257 & 759 \\
\hline
\end{tabular}

"Es el número de instituciones de salud con el mayor número de solicitudes de DP en el tema de expediente clínico.

información en el espíritu de la LFTAIPG, aún más el acceso a DP. Hasta la emisión de la Ley General de Protección de Datos Personales en posesión de los sujetos obligados (2017), se contempla la posibilidad de costo cuando implique un número mayor a 20 hojas.

2) La opción de entregar la copia en medio magnético (USB). Situación hasta el año 2017 imposible de aplicar por la limitación establecida en el Segundo Transitorio de los Lineamientos que deberán observar las dependencias y entidades de la APF en la recepción, procesamiento, trámite, resolución y notificación de las solicitudes de acceso a DP que formulen los particulares, con exclusión de las solicitudes de corrección de dichos datos. Hoy en día la Ley General de Protección de Datos Personales en posesión de los sujetos obligados contempla esa posibilidad.

3) La limitación por autoría de notas clínicas. Situación no aplicable al existir una relación laboral de los posibles autores de las notas clínicas con el Estado.

Ante esta situación se puede concluir que ninguna de las tres situaciones alegadas son aplicables a la luz de los ordenamientos legales.

Por lo anterior y con la pretensión de proporcionar alternativas a las instituciones de salud, en tanto se logre atender por un lado el cumplimiento del ejercicio del derecho y por el otro contar con la posibilidad debidamente fundamentada y motivada de limitar el acceso al mismo, se hace la siguiente propuesta (no sin antes reflexionar acerca del planteamiento inicial: la propuesta de la doble clasificación del expediente clínico).

Como ya se comentó, el soporte documental denominado expediente clínico pertenece a las instituciones de salud que en virtud de la prestación de servicios médicos genera; y por otro lado, la información contenida corresponde únicamente al titular de los datos: el paciente.

Acorde con la realidad se considera que no es conveniente que los pacientes conozcan todos los elementos e información vertida en el expediente clínico. La razón primera es que la información contenida en el mismo es en extremo sensible; lo segundo, es que resulta poco comprensible para quienes no tienen un bagaje médico, aunado a la poca utilidad que reviste para el paciente el acceso a información clínica inacabada, cuya mediana compleción resultaría fútil para cualquier propósito. Si bien, es cierto que los instrumentos normativos permiten que los datos contenidos en los expedientes clínicos puedan conocerse por el paciente o por un tercero autorizado, ello no implica que los problemas no ocurran tal y como se advirtió previamente.

Para hacer patente el problema se plantean los elementos que pueden definir las autoridades médicas de diversos centros nosocomiales:

a) Casos perinatológicos y/u obstétricos: en virtud de que para la construcción de la historia clínica se requiere atender cada uno de los elementos enunciados por la NOM 004.

b) Información que es proporcionada por el paciente al médico en aras del requerimiento de atención médica, pero que no obstante puede no ser conocida por el cónyuge y/o un tercero. Esta información 
es altamente sensible al develar información sobre vida sexual, abortos, enfermedades venéreas, entre otros.

c) Casos relacionados a salud mental en la que se involucra información de terceros. Al igual que en el inciso anterior, en aras de la atención médica requerida el paciente puede proporcionar información íntima no sólo concerniente a la esfera sexual; sino también relativa a un comportamiento de terceros y/o en vínculo, a decir: situaciones afectivas disfuncionales, maltrato infantil, abuso sexual, violencia doméstica, consumo de drogas, etc.

Consideramos, además, que el expediente clínico contiene elementos que no permiten tener una definición o conclusión definitiva o tajante respecto al estado de salud del paciente; es decir, entraña elementos no concluyentes. Ante esto estimamos que como tal, no debe darse a conocer a los pacientes, salvo que ocurriera el alta voluntaria, alta por máximo beneficio hospitalario con determinación de pronóstico ${ }^{5}$ y egreso, o alta tradicional en términos de lo referido en la NOM. Exceptuando los últimos supuestos, la información que contiene el expediente clínico no debería ser dada a conocer al paciente, en especial tratándose del pronóstico y diagnóstico, los cuales claramente se encuentran plasmados dentro del expediente clínico de manera diversa por los médicos tratantes, en virtud de que en el desempeño de sus funciones realizan anotaciones que no son definitivas o concluyentes.

Siendo un elemento fundamental de este texto el término No concluyente se abunda más sobre él. Así, vale acudir a lo que la Real Academia Española (RAE) precisa con relación al término "concluyente»: Que concluye, situación obvia y que no brinda mucha luz; pero el término «concluir» se define como: acabar o finalizar algo; y finalmente la palabra «no» que se refiere a la inexistencia, o lo contrario de lo designado por la voz a la que precede o la ausencia de lo expresado por ella. En otras palabras, debe entenderse como el evento inacabado o sin definición que trasladado a este caso implicaría en un proceso deliberativo inacabado relacionado con la salud y/o vida de una persona, en el que uno o varios servidores públicos especialmente médicos participan.

Lo anterior resulta similar con el contenido de la Ley 41/2002 básica reguladora de la autonomía del paciente y de derechos y obligaciones en materia de información y documentación clínica de España que alude al concepto «Notas subjetivas», ${ }^{5}$ el cual grosso modo implica impresiones de los profesionales sanitarios basadas en la exclusiva percepción de aquéllos.

En este orden de ideas se indicará cuáles son los momentos a los que se alude respecto a la construcción del expediente clínico relacionados con el término de notas no concluyentes:

1. Tanto el diagnóstico como el pronóstico (elementos que contiene el expediente clínico, no deben ser dados a conocer a los pacientes por no ser definitivos). Lo anterior se sostiene ante la siguiente precisión. El pronunciamiento acerca del diagnóstico clínico entendido como:

«...es el procedimiento por el cual se identifica una enfermedad entidad nosológica, síndrome o cualquier estado de salud o enfermedad (el 'estado de salud' también se diagnostica).

En términos de la práctica médica, el diagnóstico es un juicio clínico sobre el estado psicofísico de una persona; representa una manifestación en respuesta a una demanda para determinar tal estado. Diagnosticar es dar nombre al sufrimiento del paciente; es asignar una 'etiqueta'». 6

En la elaboración del diagnóstico son varios los momentos que ocurren para llegar a un razonamiento clínico y determinar la necesidad de elementos diversos que lo sustenten, situación efectiva y comprobable que se muestra en la figura 1.

En «Esta enumeración o lista de los problemas del paciente, señala la necesidad de elaborar los planes diagnósticos, que indicarán los pasos a seguir tanto en la ratificación o rectificación de la historia clínica, así

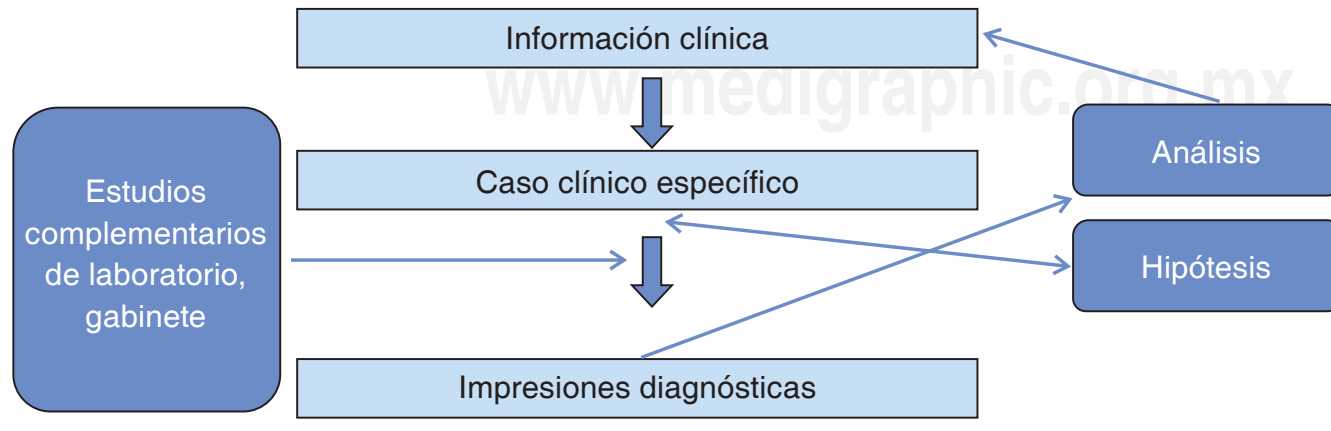

Modificado de: El razonamiento clínico como herramienta diagnóstica. ${ }^{8}$

Figura 1.

Razonamiento clínico. 
como en la orientación del planteamiento de los recursos auxiliares de laboratorio y gabinete, con tomas de productos por endoscopia rx, intervencionista u operaciones quirúrgicas.[...] Un diagnóstico exige apoyarlo en los datos obtenidos de la historia clínica. La forma más sencilla y lógica es que recurrir a los síndromes involucrados en los problemas se considere obligatorio, pues de su análisis se extraerán los que tienen actualidad, son los activos, los que desde que aparecieran continúan presentes, ya sea sin interrupción o en formas de recidivas». 6

Esta actividad queda documentada en las notas de ingreso, evolución e interconsulta, de esa suerte se entiende que en ellas se asientan los primeros hallazgos de ese análisis cognitivo que el médico entreteje para llegar a esa primera definición del proceso de atención médica.

«La integración de los diagnósticos es parte de la culminación de la historia clínica y del expediente clínico». ${ }^{6}$

Este bosquejo o presunción diagnóstica requiere agotar nuevos elementos con la intención de formular una hipótesis que seguirá siendo actualizada en el expediente como un elemento inconcluso, pues no reúne las características correspondientes para ser un diagnóstico final. Esta idea se clarifica como sigue:

«La elaboración de hipótesis es la elaboración de los síndromes a partir de los síntomas, (...) hasta que la definición del síndrome sea más acabada, pues involucra en su estructura los datos de interrogatorio, exploración física, laboratorio, gabinete, con implicaciones en el planeamiento de pronósti$\cos \gg .6$

2. El asentamiento del pronóstico clínico. Para introducirnos a este tema es importante primero definir el concepto de pronóstico. En ese sentido atenderemos a lo definido por la RAE que indica: «pronóstico es 'predecir algo futuro a partir de indicios'».

A partir de esta primera y única acepción plasmada en el diccionario de la RAE podemos asimilarlo al ámbito clínico y entender que este concepto es la predicción sobre el curso de la enfermedad en relación con la calidad de vida del paciente, nacida del engranaje de hallazgos clínicos. Esto último permite el ajuste de la concepción relativa al expediente clínico, al tratarse de un elemento natural de acuerdo a la NOM 004; pero que en muchos casos no se asienta en el documento final ${ }^{6}$ llegando a generar confusión en la expectativa de la enfermedad.
«Realizando el diagnóstico, se plantea el pronóstico, no sólo para saber el estado actual y el pasado del paciente, sino también su futuro.

El pronóstico, por tanto, ha sido una preocupación del médico que comparte con los pacientes, pues ambos están interesados en su futuro. Para establecer un pronóstico para la vida y el órgano o aparato, es necesario conocer la edad del paciente, el estado funcional en general y de los órganos involucrados, las alteraciones anatómicas, macro y microscópicas, las características de su historia clínica en sus operaciones generales y en particular sobre un paciente en especial con sus implicaciones familiares, culturales, sociales, laborales, de carga económica y los tratamientos que involucran la educación del paciente y su familia.

Es conveniente planear y justificar el pronóstico, tanto para la vida como para el órgano». ${ }^{7}$

El pronóstico es como hemos visto, el asentamiento predictivo que dirige las nuevas formas de adaptación a la enfermedad o de eventuales recaídas, que entrañan una visión futura acerca de la calidad de vida del paciente y logran una predicción respecto al funcionamiento del órgano o tejido de interés.

«El pronóstico médico es un juicio de orden racional respecto a los cambios que pueden sobrevenir en el transcurso de una enfermedad y sobre la duración y término de la misma».7

El punto de controversia estriba en que parece que el concepto está delicadamente hilado al concepto de «vida» y «muerte», en virtud de que todo índice predictivo maneja probabilidades que permiten modificar o acatar el estado en que se encuentra el paciente.

Por lo tanto, los pronósticos deben en su aplicación sujetar y cuidar lo más posible estas expectativas, apoyándose en una realidad demostrada. Para establecer un pronóstico se debe evitar el riesgo (muy frecuente) de hacer una predicción mediante la adivinación que lo que pretende más que conocer, es determinar el futuro para que este suceda como se interpreta.

«Los riesgos contemplados para la elaboración del pronóstico se conocen en medicina como morbimortalidad. El que suceda uno u otro y se reconozca como sucedido no es, no debe ser ni puede considerarse inhabilidad o negligencia, su ignorancia sí»? ${ }^{7}$ 
Para pacientes en estado terminal, el margen de error puede aumentar para el establecimiento del diagnóstico y pronóstico pudiendo ir en dos vertientes: 1) establecer un diagnóstico y pronóstico a un paciente que aún está en tratamiento, o 2) dar un diagnóstico y pronóstico a un paciente en la que ocurre el deceso antes de lo esperado. El primer caso obliga al replanteamiento diagnóstico y terapéutico; y para el segundo de los supuestos, llega a los familiares con una inesperada necesidad de procesar el duelo.

Cuando se trata de enfermedades cronicodegenerativas es mucho más evidente que un diagnóstico no ocurrirá, pues se sabe que tales enfermedades modifican su comportamiento de un momento a otro. Luego entonces se puede decir que los expedientes clínicos sin una fase conclusiva, sin el asentamiento del pronóstico, no son cerrados; esto es, no son concluyentes.

Tanto la determinación del diagnóstico como el asentamiento del pronóstico, ambos clínicos, hallan su expresión también en el proceso de atención médica de que se trate, a decir: los pacientes atendidos por consulta externa, urgencias u hospitalización. Por lo que hace a los expedientes de pacientes que se atienden únicamente por consulta externa, puede afirmarse que la información contenida en los mismos se encuentra en una primera fase de construcción. Esto es, la elaboración de las notas iniciales e historia clínica que de acuerdo a la NOM deben existir para el seguimiento médico y administrativo para la determinación del diagnóstico clínico y, en su caso, de la evolución de la enfermedad. En la mayoría de las instituciones médicas se comienza con una cita de primera vez donde se identifica a partir de la historia clínica, los signos y síntomas que proporciona el paciente y una serie de estudios de gabinete y laboratorio que apoyarán la presunción diagnóstica.

«Terminada nuestra historia clínica estamos en posibilidad de plantear qué exámenes de laboratorio o gabinete apoyan nuestro razonamiento clínico, o usan las hipótesis planteadas, con la finalidad de integrar nuestros diagnósticos e iniciar la información en una hoja de problemas.

La hoja de problemas es fundamental en el expediente médico, ahí se agrupan éstos en problemas activos e inactivos se puede decir que los problemas activos son los que integran el padecimiento actual y los inactivos son los antecedentes que han perdido actualidad». 6

Para resumir, diremos que en el expediente clínico se esbozan criterios médicos y presunciones respec- to de algún padecimiento o sospecha clínica que en algunos casos o momentos de la práctica clínica no son conclusivos. Por tanto, se actualiza el supuesto de clasificación como reservada. En este mismo orden de ideas, valga mencionar que en el expediente clínico obran anotaciones diversas concernientes a la vida íntima del propio paciente o de terceras personas, en las que debería de limitarse el acceso a terceros aún autorizados.

Un supuesto más para la limitación del acceso a la información que obra en el expediente clínico se basa en los principios de la bioética que arguyen la autonomía, justicia, beneficencia y no maleficencia bajo los cuales habrá que custodiar el proceso de atención médica. En este sentido, es necesario el apego al primero de ellos, el de la autonomía del paciente que le otorga la facultad de autogobernarse. El paciente tendría derecho a conocer o no de su información, incluso la clínica (el derecho a no saber) y a no dar a conocer información sobre su estado de salud a terceros, trátese del momento clínico de atención hospitalaria o incluso a determinar y documentar la decisión respecto a la información que obra en él posterior al fallecimiento.

Este aspecto podría fortalecer el argumento de negativa de acceso por reserva en el marco mismo de los derechos humanos, los principios de bioética y los instrumentos internacionales a los que México esté adherido, analizado a la luz del principio propersona.

\section{CONCLUSIONES}

La doble clasificación del expediente clínico debe ser aplicada para todos aquellos expedientes clínicos no concluyentes, a decir:

a) Fase de construcción (consulta externa, hospitalización).

b) Determinar la fase de razonamiento clínico y existencia de diagnóstico y/o pronóstico para determinar la conclusión del instrumento con la participación de los comités de Transparencia y de Evaluación del expediente clínico.

c) Valorar la determinación del médico tratante como estado de necesidad terapéutica para aquellos casos en los que aplique el supuesto y documentarlo.

d) Considerar la reserva de la información hacia terceros, aun cuando se trate de un expediente clínico concluido, en virtud de la sensibilidad de la información.

e) Respetar la autonomía del paciente como titular de DP para proporcionar información a terceros. 
Sin ser óbice lo anterior, se propone que el personal médico documente mediante constancia razonada dichas anotaciones subjetivas o no concluyentes o por determinación del paciente.

Respecto a la justificación legal de esta clasificación, el daño presente y probable respecto al conocimiento del pronóstico, diagnósticos y/o notas no concluyentes se comprenderá al entender que pudieran existir perjuicios, como la percepción equívoca por parte del paciente, los familiares y/o cualquier otro que tenga o no conocimiento médico; o bien de aquél que con conocimiento médico, pero con falta de ética se aproveche de esa situación inacabada para actuar en su beneficio y no del paciente.

Como corolario de lo expuesto tenemos que el acceso al expediente clínico y la información contenida en el mismo procedería, salvo las excepciones expuestas, no debe entregarse, ni darse a conocer a terceros a menos que la solicitud sea realizada por autoridad judicial o ministerial, tomando en consideración que la información contenida en él siempre tendrá el carácter de confidencial y de reservado cuando se actualicen los supuestos aquí planteados para lo cual aplicaría las dos.

\section{REFERENCIAS}

1. Ruiz MJF. Derechos constitucionales de contenido programático. Fecha de consulta: 27-I-2017. Accesible en: http://biblio.juridicas. UNAM. mx/libros /2/ 898/6.pdf

2. Carpizo MGJ. La reforma política mexicana de 1977. Fecha de consulta: 27-I-2017. Accesible en: http://biblio. juridicas. UNAM mx/libros/5/2107/5.pdf

3. Plan Nacional de Desarrollo 2013-2018. México: Presidencia de la República. Fecha de consulta: 27-I2017. Accesible en: http://www.presidencia.gob.mx

4. Oleada ER. La importancia del expediente clínico. Rev Hosp Jua Mex 2001;68:95-96.

5. Moreno MF, Moreno CA, Herrmann F, Tesse R, Girón F, Muñoz A. Anotaciones subjetivas en la historia clínica. Divergencias entre el derecho y la medicina. Boletín SPAO 2010;4(3):209-214.

6. Surós BA, Surós BJ. Semiología médica y técnica exploratoria. 8va ed. Madrid: Elservier; 2001.

7. Soberón G, Feinholz D. Muerte digna. Una oportunidad real. México, DF: Comisión Nacional de Bioética; 2008.

8. El razonamiento clínico como herramienta diagnóstica. Fecha de consulta: 01-II-2017. Accesible en http:// vitae. ucv.ve/?module $=$ articulo $\& r v=95 \& n=4205 \& \mathrm{~m}=$ $4 \& \mathrm{e}=4219$

\section{BIBLIOGRAFÍA RECOMENDADA}

- Convención Americana de Derechos Humanos. Fecha de consulta: 30-I-2017. Accesible en: http://www.juridicas. unam.mx/publica/librev/ rev/derhum/cont/22/pr/pr19. pdf

- Rodríguez ZJ. Estado y transparencia: un paseo por la filosofía política. Fecha de consulta: 30-I-2017. Accesible en: http://inicio.ifai.org.mx/Publicaciones/ Cuadernillo\%2004\%20B.pdf

- Compilación de instrumentos internacionales sobre protección de la persona aplicables en México. Fecha de consulta: 30-I-2017. Accesible en: https://www.scjn.gob.mx/ libro/instrumentos convencion/ pag 0259 .pdf pag. 180

- Constitución Política de los Estados Unidos Mexicanos. Fecha de consulta: 30-I-2017. Accesible en: http://www. diputados. gob.mx/ LeyesBiblio/htm/1.htm

- Covenio 108 del Consejo de Europa. Fecha de consulta: 30-I-2017. Accesible en: http://inicio.ifai. org.mx/Estudios/B.28-cp--CONVENIO-N-10--108-DELCONSEJO-DE-EUROPA.pdf

- Arellano MJ. Una propuesta de calidad para el manejo e integración del expediente clínico (tesis). Ciudad de México: UNAM; 2007.

- Ley General de Transparencia y Acceso a la Información Pública. México: Instituto Nacional de Acceso a la Información Pública Gubernamental, 2015. Fecha de consulta: 30-I-2017. Accesible en: http://www. diputados.gob.mx/LeyesBiblio/pdf/LGTAIP.pdf

- Ley Federal de Transparencia y Acceso a la Información Pública. México: Instituto Nacional de Acceso a la Información Pública Gubernamental; 2016. Fecha de consulta: 30-I-2017. Accesible en: http://dof. gob.mx/nota_detalle.php?codigo $=5436283 \& \mathrm{fec}$ ha $=09 / 05 / 201 \overline{6}$

- Ley Federal de Transparencia y Acceso a la Información Pública Gubernamental. México: Instituto Federal de Acceso a la Información Pública Gubernamental. 2da ed. 2002. Fecha de consulta: 30-I-2017. Accesible en: http://inicio.ifai.org.mx/LFTAIPG/LFTAIPG.pdf,

- Reglamento de la Ley Federal de Transparencia y Acceso a la Información Pública Gubernamental. México: Instituto Federal de Acceso a la Información Pública Gubernamental; 2003. Fecha de consulta: 30I-2017. Accesible en: http://www.diputados.gob.mx/ LeyesBiblio/regley/Reg_LFTAIPG.pdf,

- Ley General de Protección de Datos Personales. México: Instituto Nacional de Acceso a la Información Pública Gubernamental; 2017. Fecha de consulta: 30-I-2017. Accesible en: http://dof.gob.mx/nota_detalle.php?cod igo $=5150631 \&$ fecha $=05 \% 2$ F07\%2F 2010

- Ley General de Salud. México: Secretaría de Salud; 1984. Fecha de consulta: 30-I-2017. Accesible en: http://www. salud.gob.mx/unidades/cdi/legis/lgs/LEY_GENERAL_DE_ SALUD.pdf

- Reglamento de la Ley General de Salud en Materia de Prestación de Servicios de Atención Médica. Fecha de consulta: 30-I-2017. Accesible en: http://www. diputados.gob.mx/LeyesBiblio/regley/Reg_LGS_MP.pdf

- Norma Oficial Mexicana para el Expediente Clínico 004 SS3-2012. Fecha de consulta: 30-I-2017. Accesible en: http://dof.gob.mx/nota_detalle.php?codigo $=5272787$ \&fecha $=15 / 10 / 2012$ 
- Diario Oficial de la Federación. Lineamientos de protección de datos personales. DOF 2005 septiembre 30; primera sección: 16. Fecha de consulta: 30-I-2017. Accesible en: http://www.dof.gob.mx/nota_detalle.ph p? codigo $=5284966 \&$ fecha $=17 \% 2 F 01 \% 2 F 2013$

- Diario Oficial de la Federación. Lineamientos generales para la clasificación y desclasificación de la información de las dependencias y entidades de la Administración Pública Federal. DOF 2003 agosto 18: 31-32. Fecha de consulta: 30-I-2017. Accesible en: http://dof.gob.mx/nota detalle.php? codigo $=692002$ \&fecha $=18 / 08 / 2003$
- El derecho a saber y la instrumentación de las políticas de salud (conferencia). Semana Nacional de la Transparencia 2008. México: IFAl; 2008.

\section{$\triangle$ Correspondencia:}

Mtra. Juana Arellano-Mejía

Unidad de Transparencia, Instituto Nacional de Enfermedades Respiratorias Ismael Cosío Villegas.

Calzada de Tlalpan Núm. 4502, Colonia Sección

XVI, Del. Tlalpan, Ciudad de México.

Teléfono: 54871700 , ext. 5234

Correo electrónico: arellano@iner.gob.mx

Los autores declaran no tener conflicto de intereses. 